\title{
IAC 60 CENTENÁRIO E IAC 162 TUIUIÚ: CULTIVARES DE TRIGO PARA SEQUEIRO E IRRIGADO NO ESTADO DE SÃO PAULO ( $\left.{ }^{1}\right)$
}

\author{
JOÃO CARLOS FELÍCIO $(2,6)$, CARLOS EDUARDO DE OLIVEIRA CAMARGO $(2,6)$, \\ ANTONIO WILSON PENTEADO FERREIRA FILHO $(2,6)$, PAULO BOLLER GALLO $\left({ }^{3}\right)$, \\ VALDIR JOSUE RAMOS $\left({ }^{4}\right)$ e POLICARPO VITTII $(5,6)$
}

\begin{abstract}
RESUMO
Os cultivares de trigo IAC 60 (Centenário) e IAC 162 (Tuiuiú), provenientes de cruzamentos artificiais e obtidos por seleção genealógica, foram avaliados quanto à produção de grãos e às reaçōes aos agentes causais da ferrugem-do-colmo (casa de vegetação), da ferrugem-da-folha, da helmintosporiose e da brusone na região do Vale do Paranapanema, em cundições de sequeiro, e na região Norte do Estado de São Paulo, com irrigação por aspersão. Foram também avaliadas as qualidades industriais de panificação. As produções de grãos dos novos cultivares (sequeiro) não diferiram dos cultivares controles BH 1146 e IAC 24, em solos com elevada acidez (AA), e do Anahuac, em solos mais férteis (BA). Com irrigação, o IAC 60 e o IAC 162 expressaram todo 0 seu potencial produtivo, diferindo significativamente das testemunhas IAC 24 e Anahuac. O 'IAC 60' demonstrou moderada suscetibilidade à helmintosporiose, suscetibilidade à ferrugem-da-folha e moderada resistência à brusone, enquanto o 'IAC 162' foi resistente à ferrugem-da-folha e à brusone, porém suscetivel à helmintosporiose. O 'IAC 60' apresentou tolerância ao alumínio tóxico $\theta$ o 'IAC 162', moderada suscetibilidade; ambos foram moderadamente suscetíveis à toxicidade de ferro e exibiram tolerância para o manganês, quando testados em soluções contendo esses elementos. Nos ensaios de panificação, os novos cultivares apresentaram valores superiores aos obtidos com pães feitos com farinha de trigo comercial (padrão). O 'IAC 162' revelou volume específico do pão superior ao 'IAC 60', mas inferior quanto às características internas (miolo) e externas (crosta).
\end{abstract}

Termos de indexação: trigo, condição de sequeiro e de irrigação, produção de grãos, resistência às doenças, características de panificaçăo.

(') Com verba suplementar do Acordo entre as Cooperativas de Produtores Rurais do Vale do Paranapanema e a Secretaria de Agricultura e Abastecimento, por intermédio do Instituto Agronômico. Recebido para publicação em 10 de dezembro de 1990 e aceito em 18 de setembro de 1991.

(2) Seção de Arroz e Cereais de Inverno, Instituto Agronômico (IAC), Caixa Postal 28, 13001 Campinas (SP).

$\left(^{3}\right)$ Estação Experimental de Mococa (IAC).

(4) Estação Experimental de Itararé (IAC).

(5) Seção de Cereais, Farinhas e Panificação, Instituto de Tecnologia de Alimentos (ITAL), Caixa Postal 139, 13001 Campinas (SP).

(6) Com bolsa de pesquisa do CNPq. 


\section{ABSTRACT \\ IAC 60 CENTENÁRIO AND IAC 162 TUIUIÚ: WHEAT CULTIVARS FOR UPLAND AND IRRIGATED CONDITIONS IN THE STATE OF SÃO PAULO, BRAZIL}

In trials carried out in upland condition at the Paranapanema Valley, and under sprinkler irrigation at the North Region State of São Paulo, Brazil, the wheat cultivars IAC 60 Centenário and IAC 162 Tuiuiú originated from artificial crosses and selected by the pedigree method, were evaluated in relation to grain yield and reactions to the causal agents of leaf rust, blast, and leaf spot. Under greenhouse conditions, both cultivars were evaluated to the causal agent of the stem rust. Their industrial bread qualities were also assessed. In upland condition, using highly acid soils, the grain yield of the new cultivars did not differ from the cultivars BH 1146 and IAC 24. However, in upland conditions, but using soils with low acidity, there was no significant difference as compared to the control Anahuac. Under irrigation the cultivar IAC 60 and IAC 162 showed high grain yield, differing significantly of the controls BH 1146 and IAC 24. Considering the two regions, the cultivar IAC 60 showed moderate susceptibility to leaf spot, leaf rust and blast, while the cultivar IAC 162 exhibited resistance to leaf rust and blast, but susceptibility to leaf spot. Concerning their response to toxic aluminium, the cultivar IAC 60 was tolerant while IAC 162 showed moderate susceptibility. Both cultivars were moderately susceptible to iron toxicity and tolerant to manganese toxicity when they were tested in nutrient solutions with these elements. Considering bread characteristics trials, the new cultivars presented superior values in relation to the breads made with commercial flour. 'IAC 162' showed superior specific volume in comparison with 'IAC 60', but inferior in relation to intern (crumb) and extern (crust) characteristics.

Index terms: wheat crop, upland condition, sprinkler irrigation condition, grain yield, disease resistance, bread characteristics.

\section{INTRODUÇÃO}

Otrabalho de melhoramento genético visando à criação de novas variedades de trigo é demorado. Inicialmente, cabe investigar quais as melhores variedades diante das diferentes exigências locais de cultivo, como época de plantio, resistência às moléstias e pragas, produtividade, qualidades industriais do grão para o moinho, panificação, etc. Exige conhecimento especializado para se distinguir, entre as variedades em estudos, as que devem ser cruzadas para a possível obtenção de novos genótipos adequados às condições locais (TEIXEIRA, 1958).

Em geral, os caracteres quantitativos são os mais importantes no que diz respeito aos aspectos agronômicos. A maioria desses caracteres tem influência direta ou indireta sobre o caráter rendimento de grãos. Conseqüentemente, a identificação de um genótipo que revele potencial genético de grande produtividade passa a ser uma das tarefas mais árduas ao melhorista de trigo (CARVALHO, 1982). 
DUARTE (1974) dividiu o desenvolvimento da pesquisa de trigo no Brasil em quatro etapas: a primeira, a menos importante, descreve o início da pesquisa e se estende até por volta de 1940; a segunda inclui trabalhos de duas décadas (1940-60), podendo ser determinada com o lançamento do famoso trigo precoce 'Frontana', de adaptação regional ampla e extrema rusticidade; o lançamento do trigo IAS 20, em 1963, marca a terceira etapa, e a quarta etapa inicia-se nos últimos anos da década de sessenta. $O$ aumento do estoque de conhecimento cientffico e o próprio desenvolvimento da triticultura implicaram a modificação do planejamento da pesquisa e, até mesmo, a metodologia dos programas fitossanitários, que foram melhorados e ampliados a varias doenças, havendo preocupação também com a fertilidade do solo e com a qualidade industrial do produto.

FELÍClO et al. $(1983,1985,1988,1990)$, mediante trabalhos de melhoramento genético, selecionaram novos cultivares de trigo no Instituto Agronômico de Campinas, mais adaptados às condições de clima e solo da região triticola situada ao norte do paralelo $24^{\circ} \mathrm{S}$., destacando-se IAC 18 (Xavantes), IAC 21 (Iguaçu), IAC 24 (Tucurun), este de porte semi-anão e com tolerância ao alumínio tóxico e, mais recentemente, IAC 25 (Pedrinhas) e IAC 161 (Taiamã).

O presente trabalho tem por objetivo estudar o comportamento de dois novos cultivares de trigo, IAC 60 (Centenário) e IAC 162 (Tuiuiú) nas condições de sequeiro e de irrigação por aspersão, no Estado de São Paulo.

\section{MATERIAL E MÉTODOS}

O cultivar IAC 60 (Centenário) foi obtido pelo método genealógico (ALLARD, 1960); provém de seleção do híbrido 1196, originário do cruzamento entre os cultivares IRN 33-70 e IAC 5, sendo o primeiro oriundo do Estado de Dakota do Norte-EUA, com a denominação de 'Kurtzman', M12, proveniente do "International Spring Wheat Rust Nursery" - 1970, e o cultivar IAC 5 Maringá resultou do cruzamento Frontana/Kenya 58//Ponta Grossa I (CAMARGO, 1972). O cultivar Centenário apresenta as seguintes características: altura entre 80 e $90 \mathrm{~cm}$, ciclo médio de 125-130 dias da emergência à colheita, aurícula verde-amarelado-clara; folha pendente verde-escura e espiga de coloração creme, podendo apresentar algumas avermelhadas, aristada e oblonga, com comprimento aproximado de $9,5 \mathrm{~cm}$ e grão ovóide e creme (Figura 1A).

O cultivar IAC 162 (Tuiuiú) é uma linhagem selecionada no Instituto Agronômico, Campinas, proveniente do híbrido Kavkaz/CIANO 67/Penjamo 62, originário do Centro Internacional de Melhoramento de Milho e Trigo (CIMMYT) - México. 0 'Tuiuiú' apresenta as seguintes características: altura de plantas entre $70 \mathrm{e} 80 \mathrm{~cm}$, ciclo precoce, de 120 a 125 dias da emergência à maturação, hábito vegetativo ereto, aurícula verde-amarelado-clara, folhas verde-escuras, de posição intermediária,e espiga creme, aristada e fusiforme, com comprimento aproximado de 8,5 a $9,5 \mathrm{~cm}$, e grão avermelhado (Figura 1B). 
Foram instalados, em 1985-89, no Vale do Paranapanema (oeste do Estado de São Paulo), em condições de sequeiro, experimentos com o objetivo de avaliar o comportamento de cultivares de trigo nas localidades de São José das Laranjeiras, Cândido Mota, Maracal e Cruzália. Desses ensaios foram extraídos os dados dos cultivares IAC 60 e IAC 162 e comparados com os dos cultivares comerciais mais produtivos em semeadura na região: $\mathrm{BH} 1146$, Anahuac e IAC 24. Cada parcela foi constituída de cinco linhas de $5 \mathrm{~m}$ de comprimento, espaçadas de $0,20 \mathrm{~m}$, com separação entre as parcelas de $0,60 \mathrm{~m}$.

Nas áreas de irrigação por aspersão, instalaram-se experimentos para avaliar o comportamento dos cultivares IAC 60 e IAC 162, comparados aos cultivares comerciais Anahuac e IAC 24, nas localidades de Guaíra, Colômbia, Mococa, Pirassununga, Aguaí, Tatuí e Campinas, em 1987-89. Adotou-se procedimento semelhante ao utilizado nos experimentos de sequeiro.

Anteriormente à instalação dos ensaios, retiraram-se amostras compostas dos solos cujas análises foram efetuadas na Seção de Fertilidade do Solo e Nutrição de Plantas do Instituto Agronômico, visando a sua classificação quanto à fertilidade. Os experimentos receberam adubação a lanço constante de $20 \mathrm{~kg}$ de $\mathrm{N}, 60 \mathrm{~kg}$ de $\mathrm{P}_{2} \mathrm{O}_{5}$ e $20 \mathrm{~kg}$ de $\mathrm{K}_{2} \mathrm{O}$, por hectare, nas formas de sulfato de amônio, superfosfato simples e cloreto de potassio.

Para o estudo do comportamento dos cultivares em relação à ferrugem-do-colmo, Puccinia graminis f. sp. tritici, avaliaram-se plântulas no Centro Nacional de Pesquisa de Trigo, de acordo com os critérios do quadro 4. Utilizaram-se os cultivares $\mathrm{BH} 1146$ e IAC 5 como controle nos testes de reação à ferrugem-do-colmo em casa de vegetação. A ferrugem-da-folha, Puccinia recondita f. sp. tritici., foi avaliada em plantas adultas em condições de campo, mediante a escala modificada de Cobb, empregada no "International Rust Nursery" e utilizada por SCHRAMM et al. (1974): é composta por um número estimando a porcentagem de ataque de moléstia no colmo e/ou na folha. Para outras moléstias, como Cochliobolus sativus (forma imperfeita de Helminthosporium sativum Pam., King \& Bakke), ocorrentes nas folhas, as informações foram estimadas em porcentagem de área foliar infectada, observando-se as folhas superiores e, para a brusone do trigo, Pyricularia sp.,as avaliações foram efetuadas nas folhas, principalmente nas espigas (IGARASHI, 1986).

A altura de planta de cada cultivar foi medida levando-se em consideração a distância do nível do solo ao ápice da espiga, mantendo-se as plantas esticadas e excluindo-se as aristas.

Os cultivares IAC 60 e IAC 162 foram testados para tolerância a 0, 2, 4, 6, 8 e $10 \mathrm{mg} /$ litro de $\mathrm{Al}^{3+}$, mantendo-se constante a temperatura de $25^{\circ} \mathrm{C}$ (CAMARGO et al., 1987); para a tolerância a 0,11;300;600 e 1.200mg/litro de Mn $^{2+}$ (CAMARGO et al., 1983) e para tolerância a 0,$56 ; 5 ; 10 ; 20$ e $40 \mathrm{mg}$ de $\mathrm{Fe}^{2+}$ (CAMARGO et al., 1988), empregando-se soluções nutritivas. Em todos os testes, usaram-se como controle os cultivares $\mathrm{BH} 1146$ (tolerante à toxicidade de $\mathrm{Al}^{3+}$ e sensível à toxicidade de $\mathrm{Mn}^{2+}$ e Fe ${ }^{2+}$ ) e Siete Cerros (sensível à toxicidade de $\mathrm{Al}^{3+}$ e tolerante à toxicidade de $\mathrm{Mn}^{2+}$ e $\mathrm{Fe}^{2+}$ ). 

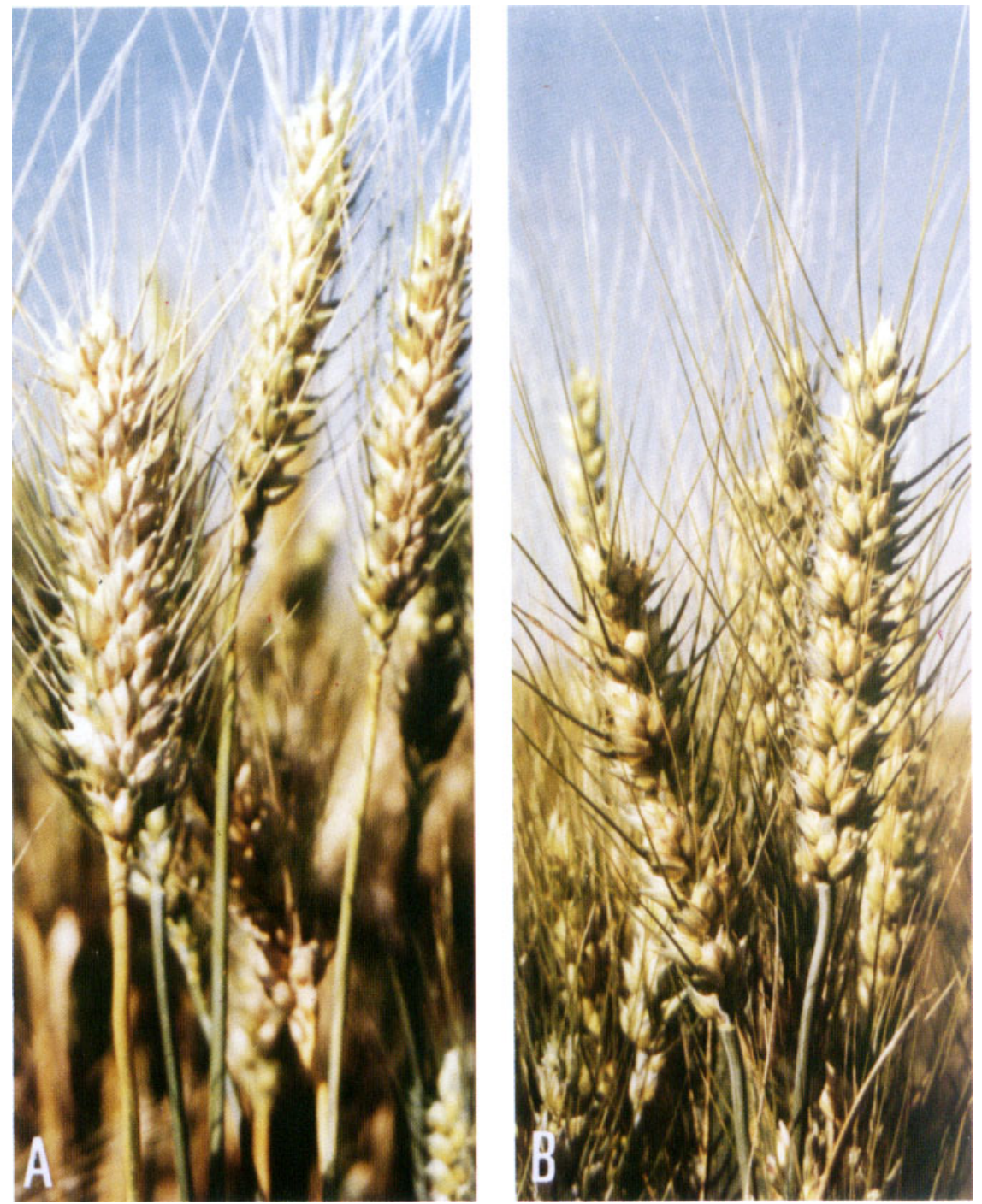

FIGURA 1. A: Espigas características do cultivar IAC-60, Centenário, apresentando a mesma espessura do centro ao ápice: aristada, em fase de granação. B: Espigas características do cultivar IAC-162; Tuiuiú, fusiforme, afinando do centro ao ápice: aristada, em fase de granação. 
Para a irrigação, adotou-se o método proposto por SILVA et al. (1984): consiste em uma irrigação de $40-60 \mathrm{~mm}$ após a semeadura, com a finalidade de umedecer o solo, bem como a instalação de tensiômetros, em pontos diferentes, à profundidade de $12 \mathrm{~cm}$. As irrigações complementares foram realizadas quando a média das leituras dos tensiômetros apresentava 0,6 atm., e a lâmina liquida aplicada foi determinada através da evaporação no tanque classe $A$, entre intervalos das irrigações.

Os grãos de trigo dos cultivares IAC 60 e IAC 162 foram submetidos a teste de moagem, para determinação das características de rendimento de farinha. Utilizou-se para isso o moinho Buhler, modelo MLU-202, trabalhando-se com $5 \mathrm{~kg}$ de grãos para cada moagem. Os grãos tiveram sua umidade ajustada de acordo com o método oficial ARBEITSGEMEINSCHALT (1971). Na moagem, as características obtidas foram em farinha, farelo grosso e farelo fino.

Essas farinhas foram submetidas a análises de ordem física no farinógrafo, amilógrafo e extensógrafo, conforme método da AMERICAN ASSOCIATION OF CEREAL CHEMISTS(AACC) (1969). O teor e a qualidade do glúten e o 'falling-number" foram determinados segundo BÄR (1982). O expansograma foi determinado segundo o método oficial ARBEITSGEMEINSCHALT (1971).

Para os testes de panificação, usou-se a formulação descrita por VITTI et al. (1982). Preparou-se o pão do tipo francês de 80 gramas de massa por unidade, segundo descrito por VITTI et al. (1980). Compararam-se os pães com os preparados com farinha comercial, sendo a avaliação efetuada de acordo com VITTI \& PIZZINATTO (1975).

\section{RESULTADOS E DISCUSSÃO}

A análise estatística conjunta dos ensaios efetuados em condição de sequeiro revelou efeitos significativos para todas as causas da variação em estudo Quadro 1. Analisando-se os experimentos de acordo com a fertilidade do solo em que foram realizados, verificou-se a não-ocorrência de significância para cultivar e para os diferentes tipos de solo (baixa ou alta fertilidade) - Quadro 2.

Os resultados obtidos nos experimentos efetuados em condição de sequeiro, com os cultivares BH 1146 e IAC 24 como testemunhas para solos de baixa fertilidade natural (BF) das localidades de Cândido Mota e Maracaí e o 'Anahuac', para solos com alta fertilidade (AF), São José das Laranjeiras e Cruzália para comparação com os cultivares IAC 60 e IAC 162, de 1985 a 1989, no Vale do Paranapanema, encontram-se no quadro 2.

Mesmo não havendo diferenças significativas dos cultivares controles em condição de sequeiro, os novos cultivares IAC 60 e IAC 162 demonstraram boa adaptabilidade regional, pela similaridade de comportamento com aqueles em cultivo utilizados como testemunha, independente da fertilidade do solo na qual foram avaliados. 
Quadro 1. Quadrados médios da análise da variância para rendimento de grãos de cultivares de trigo em condição de sequeiro no período 1985-89 e com irrigação por aspersão em 1987-89

Causas da

Sequeiro

Irrigado

variação

\begin{tabular}{ll}
\multicolumn{2}{c}{ Sequeiro } \\
\hline GL & Quadrado médio
\end{tabular}

Origado

GL Quadrado médio

\begin{tabular}{lrrrr} 
Ano & 4 & $18.934 .275,80^{*}$ & 2 & $78.618 .731,93^{*}$ \\
Cultivar & 4 & $793.796,75$ & 3 & $4.646 .327,46^{*}$ \\
Local & 3 & $5.348 .321,72^{*}$ & 6 & $11.317 .943,84^{*}$ \\
Ano x cultivar & 16 & $734.632,16^{*}$ & 6 & $977.985,57^{*}$ \\
Ano x local & 12 & $4.485 .355,26^{*}$ & 12 & $6.161 .551,92^{*}$ \\
Cultivar x local & 12 & $414.993,46^{*}$ & 18 & $440.227,43^{*}$ \\
Resíduo & 348 & $154.006,14$ & 288 & $371.152,91$ \\
\hline
\end{tabular}

* Significativo ao nível de $5 \%$.

QUADRO 2. Produções médias de grãos dos cultivares de trigo obtidas nos ensaios realizados em condição de sequeiro em solos considerados de baixa (BF) e alta fertilidade (AF), no período 1985-89

\begin{tabular}{|c|c|c|c|c|c|c|}
\hline Cultivares & $\begin{array}{c}\text { São José das } \\
\text { Laranjeiras } \\
\text { AF }\end{array}$ & $\begin{array}{c}\text { Cruzália } \\
\text { AF }\end{array}$ & Média & $\begin{array}{c}\text { Maracaí } \\
\text { BF }\end{array}$ & $\begin{array}{l}\text { Cândido } \\
\text { Mota } \\
\text { BF }\end{array}$ & Média \\
\hline $\begin{array}{l}\text { BH } 1146 \\
\text { IAC } 60 \\
\text { IAC } 162 \\
\text { IAC } 24 \\
\text { Anahuac } \\
\text { F (local) }\end{array}$ & $\begin{array}{l}2405 a \\
2074 b \\
2059 b \\
1984 b \\
2215 a b\end{array}$ & $\begin{array}{l}2232 a \\
2288 a \\
2060 a \\
2244 a \\
2126 a\end{array}$ & $\begin{array}{c}2319 a \\
2181 a \\
2060 a \\
2114 a \\
2171 a \\
11,83 n s\end{array}$ & $\begin{array}{l}1943 a \\
1856 a b \\
1666 b \\
1637 b \\
1321 c\end{array}$ & $\begin{array}{l}2037 a \\
2112 a \\
2181 a \\
2057 a \\
1930 a\end{array}$ & $\begin{array}{c}1990 \mathrm{a} \\
1984 \mathrm{a} \\
1924 \mathrm{a} \\
1847 \mathrm{a} \\
1626 \mathrm{a} \\
3,85 \mathrm{~ns}\end{array}$ \\
\hline Média & $2151 A B$ & $2190 A$ & & $1685 C$ & 2064B & \\
\hline
\end{tabular}

Médias para comparação da produção de grãos entre cultivares dentro de um local, em letras minúsculas, e médias para comparação entre locais, em letras maiúsculas. Médias seguidas pela mesma letra não diferem estatisticamente entre si pelo teste de Duncan a $5 \%$ de probabilidade.

A análise estatística conjunta dos ensaios com irrigação mostrou efeitos significativos para ano, cultivar, local e para as suas interações (Quadro 1).

No quadro 3 encontram-se os dados dos ensaios com irrigação por aspersão nas localidades de Gualra, Colômbia, Mococa, Pirassununga, Agual, Tatul e Campinas, em 1987-89. 
QUADRO 3. Produções médias de grãos de trigo obtidas nos ensaios em condição de irrigação por aspersão em sete localidades paulistas no período 1987-89

\begin{tabular}{|c|c|c|c|c|c|c|c|c|}
\hline \multirow[t]{2}{*}{ Cultivar } & \multirow[t]{2}{*}{ Guaíra } & \multirow[t]{2}{*}{ Colômbia } & \multirow[t]{2}{*}{ Mococa } & \multicolumn{2}{|c|}{ Pirassununga Aguaí } & \multirow[t]{2}{*}{ Tatuí } & \multirow[t]{2}{*}{ Campinas } & \multirow[t]{2}{*}{ Média } \\
\hline & & & & - $\mathrm{kg}$ & & & & \\
\hline $\begin{array}{l}\text { IAC } 60 \\
\text { IAC } 162 \\
\text { IAC } 24 \\
\text { Anahuac } \\
\text { Média }\end{array}$ & $\begin{array}{l}4179 a \\
4233 a \\
3665 a \\
3971 a b \\
4012 A\end{array}$ & $\begin{array}{l}4255 a \\
3670 b \\
3777 a b \\
3652 b \\
3839 A\end{array}$ & $\begin{array}{l}3447 a \\
3667 a \\
2937 a \\
2875 b \\
3031 B\end{array}$ & $\begin{array}{l}3937 a \\
3487 a b \\
3190 b \\
3320 b \\
3483 B\end{array}$ & $\begin{array}{l}2873 a \\
2869 a \\
2384 a b \\
2342 b \\
2617 D\end{array}$ & $\begin{array}{l}3199 a \\
2841 a \\
2854 a \\
2886 a \\
2445 C\end{array}$ & $\begin{array}{l}3341 a \\
3608 a b \\
3300 a b \\
2884 b \\
3283 B\end{array}$ & $\begin{array}{c}3604 a \\
3482 a \\
3158 b \\
3153 b\end{array}$ \\
\hline
\end{tabular}

Médias de cultivares seguidas por diferentes letras minúsculas no sentido vertical diferem entre si, em um mesmo local, e no sentido horizontal, em letras maiúsculas, entre os locais em estudo (Duncan a 5\%).

Os cultivares IAC 60 e IAC 162 apresentaram rendimentos superiores aos dos cultivares IAC 24 e Anahuac, diferindo destes pelo teste de Duncan a 5\%. O IAC 60, em Colômbia e Guaíra, apresentou rendimento médio de 4.255 e $4.179 \mathrm{~kg} / \mathrm{ha}$ respectivamente, demonstrando o seu alto potencial de rendimento, $e$ o IAC 162 adaptou-se melhor em Guaíra em solo com melhor fertilidade.

Desde 1917 tem-se tratado em literatura cientifica da estabilidade das variedades com altos rendimentos (CIMMYT, 1985). Entretanto, algumas publicações cientfficas bastante conhecidas têm declarado erroneamente que as variedades só funcionam bem em condições quase ótimas e que fatores como a seca, enfermidades e o uso reduzido de insumos afetam o seu desempenho.

Os resultados demonstraram que os novos cultivares IAC 60 e IAC 162 apresentaram produtividade estável de grãos para as diferentes condições em relação aos controles utilizados.

As avaliações dos novos cultivares quanto à resistência a Puccinia graminis f. sp. tritici em casa de vegetação encontram-se no quadro 4. O 'IAC 60' apresentou reações idênticas ao seu genitor IAC 5, exceto para as raças G 17, G 18 e G 23, expressando resistência no estádio de plântula. O 'IAC 162' apresentou resistência às raças testadas do patógeno, sendo, portanto, superior aos demais cultivares em teste. $\mathrm{O}$ 'BH 1146' foi suscetível a todas as raças em estudo.

No Vale do Paranapanema (cultura de sequeiro), a ocorrência de Helminthosporium sp. vem prevalecendo nos últimos anos em relação às demais moléstias (FELICIO et al., 1986) - Quadro 5.

A análise estatística revelou que a porcentagem de área foliar infectada foi mais influenciada pelo ano, seguida pelo local do estudo e pela reação do cultivar. Entre os cultivares estudados, IAC 24, IAC 162 e IAC 60 apresentaram-se mais suscetiveis que Anahuac e BH 1146. 
QUADR0 4. Reações de cultivares de trigo às raças de Puccinia graminis f. sp. tritici, em casa de vegetação, no estádio de plântula

\begin{tabular}{llllllllll}
\hline \multirow{2}{*}{ Cultivar } & \multicolumn{1}{c}{ Raças de Puccinia graminis tritici } \\
\cline { 2 - 9 } & G11 & G17 & G18 & G19 & G20 & G21 & G22 & G23 & G24 \\
\hline IAC 5 & 3 & 3 & 3 & 3 & 3 & 3 & 3 & 3 & 3 \\
IAC 60 & 3 & $0 ;$ & $0 ; 1$ & 3 & 3 & 3 & 3 & $2 ; 3$ & 3 \\
IAC 162 & $0 ;$ & $1-$ & $0 ;$ & $0 ;$ & $0 ;$ & $0 ;$ & $0 ;$ & $0 ;$ & $0 ;$ \\
BH 1146 & 4 & 4 & 4 & 3 & 4 & 4 & 4 & 4 & 4 \\
\hline
\end{tabular}

Fonte: Centro Nacional de Pesquisa de Trigo.

Escala de leitura: $O$ imune: $0 ; 1$ e 2 resistente; 3 e 4 suscetível.

QUADRO 5. Reações médias em área foliar com sintomas de Helminthosporium sp., em cultivares de trigo no período $1985-89$, em condições de sequeiro, no Vale do Paranapanema

\begin{tabular}{lllllll}
\hline Cultivar & 1985 & 1986 & 1987 & 1988 & 1989 & Média \\
\hline IAC 24 & $27,5 \mathrm{a}$ & $20,0 \mathrm{a}$ & $37,5 \mathrm{a}$ & $55,0 \mathrm{a}$ & $27,5 \mathrm{a}$ & $33,5 \mathrm{a}$ \\
IAC 162 & $20,0 \mathrm{a}$ & $17,5 \mathrm{a}$ & $40,0 \mathrm{a}$ & $52,5 \mathrm{a}$ & $30,0 \mathrm{a}$ & $32,0 \mathrm{ab}$ \\
IAC 60 & $20,0 \mathrm{a}$ & $17,5 \mathrm{a}$ & $27,5 \mathrm{ab}$ & $52,5 \mathrm{a}$ & $22,5 \mathrm{a}$ & $28,0 \mathrm{ac}$ \\
Anahuac & $15,0 \mathrm{a}$ & $12,5 \mathrm{a}$ & $32,5 \mathrm{ab}$ & $30,0 \mathrm{~b}$ & $32,5 \mathrm{a}$ & $25,0 \mathrm{bc}$ \\
BH 1146 & $15,0 \mathrm{a}$ & $15,0 \mathrm{a}$ & $17,5 \mathrm{~b}$ & $32,5 \mathrm{~b}$ & $30,0 \mathrm{a}$ & $21,5 \mathrm{c}$ \\
Média & $19,5 \mathrm{C}$ & $16,5 \mathrm{C}$ & $31,0 \mathrm{~B}$ & $44,5 \mathrm{~A}$ & $28,5 \mathrm{~B}$ & \\
& & & & & & \\
F. (cultivar) & & & & & & $3,28^{*}$ \\
F. (ano) & & & & & & $5,55^{*}$ \\
F. (local) & & & & & & \\
\hline
\end{tabular}

Médias de cultivares seguidas por letras distintas no sentido vertical para cultivar $\theta$ horizontal para ano diferem entre si pelo teste de Duncan a $5 \%$.

Para a ocorrência da ferrugem-da-folha, agente causal Puccinia recondita f. sp. tritici, o fator ano influenciou diretamente na epifitia da moléstia. Os cultivares IAC 24, BH 1146 e IAC 60 foram mais suscetíveis que IAC 162 e Anahuac -Quadro 6.

Para as regiões com irrigação por aspersão - Quadro 7 - a ocorrência das moléstias foi também influenciada pelos fatores ano, local e reação do cultivar. Verificou-se a presença generalizada de helmintosporiose em todos os locais, não havendo diferença entre os cultivares. Para a ferrugem-da-folha, o Anahuac e o IAC 162 apresentaram os menores índices de infecção. 
QUADRO 6. Reações médias em área foliar com ataque da ferrugem-da-folha, agente causal Puccinia recondita f. sp. tritici, em cultivares de trigo, em condições de sequeiro, no período 1985-89 no Vale do Paranapanema

\begin{tabular}{lrrrrrr}
\hline Cultivar & 1985 & 1986 & 1987 & 1988 & 1989 & Média \\
\hline IAC 24 & $10,0 \mathrm{a}$ & $1,25 \mathrm{a}$ & $18,75 \mathrm{a}$ & 0 & $21,25 \mathrm{a}$ & $10,15 \mathrm{a}$ \\
BH 1146 & $3,8 \mathrm{a}$ & $0,00 \mathrm{a}$ & $22,50 \mathrm{a}$ & 0 & $55,50 \mathrm{a}$ & $9,75 \mathrm{a}$ \\
IAC 60 & $11,3 \mathrm{a}$ & $0,00 \mathrm{a}$ & $17,50 \mathrm{a}$ & 0 & $13,75 \mathrm{ab}$ & $8,50 \mathrm{a}$ \\
IAC 162 & $5,0 \mathrm{a}$ & $0,00 \mathrm{a}$ & $2,50 \mathrm{~b}$ & 0 & $10,00 \mathrm{bc}$ & $3,50 \mathrm{~b}$ \\
Anahuac & $2,5 \mathrm{a}$ & $0,00 \mathrm{a}$ & $3,75 \mathrm{~b}$ & 0 & $1,25 \mathrm{c}$ & $1,50 \mathrm{~b}$ \\
Média & $13,0 \mathrm{~A}$ & $0,25 \mathrm{C}$ & $13,0 \mathrm{~A}$ & $0 \mathrm{C}$ & $13,75 \mathrm{~A}$ & \\
F. (ano) & & & & & & \\
F. (cultivar) & & & & & & $15,70^{*}$ \\
F. (local) & & & & & & $5,75^{*}$ \\
\hline
\end{tabular}

Médias seguidas por letras distintas no sentido vertical para cultivar $\theta$ para ano na horizontal diferem entre si pelo teste de Duncan a 5\%. * Significativo ao nível de 5\%.

QUADRO 7. Reações médias em área foliar com sintomas de ferrugem-da-folha $e$ helmintosporiose, em culttvares de trigo estudados em condição de irrigação por aspersão no Estado de São Paulo, no período 1987-89

\begin{tabular}{|c|c|c|c|c|c|c|c|c|}
\hline \multirow{2}{*}{ Cultivar } & \multicolumn{4}{|c|}{ Ferrugem-da-folha } & \multicolumn{4}{|c|}{ Helmintosporiose } \\
\hline & 1987 & 1988 & 1989 & Média & 1987 & 1988 & 1989 & Média \\
\hline $\begin{array}{l}\text { IAC } 24 \\
\text { IAC } 60 \\
\text { Anahuac } \\
\text { IAC } 162 \\
\text { Média }\end{array}$ & $\begin{array}{c}15,7 a \\
10,0 a b \\
0,8 c \\
2,2 b c \\
7,14 A\end{array}$ & $\begin{array}{l}4,3 \mathrm{a} \\
2,9 \mathrm{a} \\
4,3 \mathrm{a} \\
2,8 \mathrm{a} \\
3,57 \mathrm{AB}\end{array}$ & $\begin{array}{l}2,2 \mathrm{a} \\
2,2 \mathrm{a} \\
0,0 \mathrm{a} \\
0,0 \mathrm{a} \\
1,07 \mathrm{~B}\end{array}$ & $\begin{array}{l}7,4 a \\
5,0 a \\
1,7 a \\
1,7 a\end{array}$ & $\begin{array}{l}31,4 a \\
20,8 b \\
19,3 b \\
23,6 a b \\
23,75 A\end{array}$ & $\begin{array}{l}17,9 a \\
13,6 a \\
13,6 a \\
16,6 a \\
15,35 B\end{array}$ & $\begin{array}{l}28,6 a \\
24,3 a \\
30,0 a \\
28,6 a \\
27,85 A\end{array}$ & $\begin{array}{c}25,9 a \\
19,5 a \\
21,0 a \\
22,9 a \\
-\end{array}$ \\
\hline $\begin{array}{l}\text { F. (ano) } \\
\text { F. (cultivar) } \\
\text { F. (local) }\end{array}$ & & & & $\begin{array}{l}4,60^{\star} \\
2,88^{\star} \\
5,78^{\star}\end{array}$ & & & & $\begin{array}{c}16,68^{*} \\
2,38 \mathrm{~ns} \\
14,33^{*}\end{array}$ \\
\hline
\end{tabular}

Médias seguidas por letras distintas no sentido vertical para cultivar e no horizontal para ano diferem entre si pelo teste de Duncan a 5\%. * Significativo ao nível de 5\%.

No quadro 8, é demonstrada a reação dos cultivares estudados a Pyricularia sp. (brusone do trigo) no Vale do Paranapanema, em condições de sequeiro, em 1988. 
QUADRO 8. Reação de cultivares de trigo à brusone em condições de sequeiro no Vale do Paranapanema, em 1988, no Estado de São Paulo

\begin{tabular}{|c|c|c|c|c|c|c|c|}
\hline \multirow[b]{2}{*}{ Culltivar } & \multicolumn{4}{|c|}{ Espigas ( $\left.{ }^{1}\right)$} & \multirow[b]{2}{*}{ Média } & \multicolumn{2}{|c|}{ Folha $\left({ }^{2}\right)$} \\
\hline & Maracaí & Cruzália & $\begin{array}{l}\text { São José das } \\
\text { Laranjeiras }\end{array}$ & $\begin{array}{l}\text { Cândido } \\
\text { Mota }\end{array}$ & & Média & Reação( $\left.{ }^{3}\right)$ \\
\hline & & & & & $\%$ & $\%$ & \\
\hline $\begin{array}{l}\text { BH } 1146 \\
\text { IAC } 60 \\
\text { IAC } 24 \\
\text { IAC } 162 \\
\text { Anahuac }\end{array}$ & $\begin{array}{l}1 \\
2 \\
1 \\
1 \\
3\end{array}$ & $\begin{array}{l}1 \\
2 \\
1 \\
1 \\
4\end{array}$ & $\begin{array}{l}1 \\
2 \\
1 \\
1 \\
4\end{array}$ & $\begin{array}{l}- \\
2 \\
- \\
- \\
2\end{array}$ & $\begin{array}{l}0,75 \\
2,00 \\
0,75 \\
0,75 \\
3,25\end{array}$ & $\begin{array}{l}20 \\
20 \\
10 \\
10 \\
40\end{array}$ & $\begin{array}{r}R \\
M R \\
R \\
R \\
S\end{array}$ \\
\hline
\end{tabular}

(1) 1 representa 0-20\%; 2: 21-40\%; 3: 41-60\%; 4: 61-80\%, e 5: 81-100\% de ocorrência da moléstia.

(2) Média da porcentagem da área foliar atacada.

(3) R: resistente; MR: moderadamente resistente; S: suscetível.

A reação dos cultivares à brusone demonstrou alta suscetibilidade do cultivar Anahuac, de origem mexicana, moderada resistência do 'IAC 60' e resistência para os cultivares $\mathrm{BH} 1146$, IAC 24 e IAC 162.

A média do comprimento de dez raízes dos cultivares IAC 162 e IAC 60, bem como dos controles $\mathrm{BH} 1146$ e Siete Cerros, medidas após 72 horas de crescimento nas soluções nutritivas completas, que se seguiu a 48 horas de crescimento nas soluções de tratamento contendo seis concentrações de aluminio, encontram-se no quadro 9.

Em todos os cultivares, houve redução do crescimento radicular nas soluções de tratamento contendo $\mathrm{Al}^{3+}$ em relação ao das soluções de tratamento contendo $0 \mathrm{mg} /$ /itro de $\mathrm{Al}^{3+}$. O Siete Cerros revelou-se muito sensível ao alumínio tóxico, pois suas raízes primárias não cresceram em solução com $2 \mathrm{mg} /$ /itro de $\mathrm{Al}^{3+}$. O IAC 162 exibiu crescimento das raízes primárias até a concentração de $4 \mathrm{mg} /$ /itro, sendo considerado moderadamente suscetível. O IAC 60 e o BH 1146 exibiram crescimento radicular, mesmo quando testados a $10 \mathrm{mg} /$ ittro de $\mathrm{Al}^{3+}$, sendo, portanto, considerados tolerantes.

O comprimento médio das raízes dos cultivares de trigo, medido após doze dias de crescimento em soluções contendo cinco concentrações de ferro, encontra-se no quadro 10.

Os resultados confirmaram ser o $\mathrm{BH} 1146$ muito sensível à toxicidade causada por doses altas de ferro. Houve uma redução de $75 \%$ no crescimento das suas raízes, à medida que a concentração de ferro de 0,56 passou para $40 \mathrm{mg} / \mathrm{litro}$. Os cultivares IAC 162 e IAC 60 mostraram reduções de 65 e 63\% respectivamente, sendo considerados de reações intermediárias, tendendo mais para a suscetibilidade. E o 'Siete Cerros' apresentou-se como tolerante. 
QUADRO 9. Comprimento médio das raizes de cultivares de trigo, medidos após 72 horas de crescimento em soluções nutritivas completas, que se seguiu a 48 horas de crescimento nas soluções-tratamento contendo seis concentrações de alumínio, em temperatura constante de $25 \pm 1^{\circ} \mathrm{C}$

\begin{tabular}{|c|c|c|c|c|c|c|}
\hline \multirow{2}{*}{ Cultivares } & \multicolumn{6}{|c|}{ Concentrações de alumínio em mg/litro } \\
\hline & 0 & 2 & 4 & 6 & 8 & 10 \\
\hline & \multicolumn{6}{|c|}{$\mathrm{mm}$} \\
\hline IAC 162 & 65,5 & 31,3 & 4,7 & 0,0 & 0,0 & 0,0 \\
\hline IAC 60 & 72,1 & 71,1 & 38,7 & 22,3 & 16,8 & 1,5 \\
\hline BH 1146 & 95,1 & 62,6 & 48,8 & 40,0 & 32,6 & 26,6 \\
\hline S. Cerros & 62,5 & 0,0 & 0,0 & 0,0 & 0,0 & 0,0 \\
\hline
\end{tabular}

QUADRO 10. Comprimento médio das raízes de cultivares de trigo medidos após 12 dias de crescimento em soluções nutritivas contendo cinco concentrações de ferro

\begin{tabular}{lccccccccccc}
\hline \multirow{2}{*}{ Cultivares } & \multicolumn{10}{c}{ Concentrações de ferro nas soluções em mg/litro } \\
\cline { 2 - 12 } & \multicolumn{2}{c}{0,56} & \multicolumn{2}{c}{5} & \multicolumn{1}{c}{10} & \multicolumn{2}{c}{20} & & 40 \\
\hline & $\mathrm{mm}$ & $\%$ & $\mathrm{~mm}$ & $\%$ & $\mathrm{~mm}$ & $\%$ & $\mathrm{~mm}$ & $\%$ & $\mathrm{~mm}$ & $\%$ \\
IAC 162 & 130,7 & 100 & 122,8 & 94 & 91,5 & 70 & 51,3 & 39 & 45,3 & 35 \\
IAC 60 & 115,2 & 100 & 104,0 & 90 & 69,5 & 60 & 46,9 & 41 & 43,3 & 33 \\
BH 1146 & 222,6 & 100 & 199,4 & 90 & 159,1 & 71 & 68,5 & 29 & 56,1 & 25 \\
S. Cerros & 151,0 & 100 & 144,1 & 95 & 106,4 & 70 & 79,2 & 52 & 68,1 & 45 \\
\hline
\end{tabular}

Os dados obtidos das raizes dos cultivares de trigo medidos após quinze dias de crescimento em solução contendo diferentes concentrações de manganês acham-se no quadro 11.

A elevada suscetibilidade do 'BH 1146' à toxicidade de manganês em doses elevadas é confirmada pela redução de $79 \%$ no crescimento das raízes à medida que se aumentaram as concentrações de manganês nas soluções de 0,11 para $1.200 \mathrm{mg} /$ litro. Os cultivares IAC 162 e IAC 60 revelaram a mesma tolerância demonstrada pelo Siete Cerros, com redução no sistema radicular de 52, 43 e 47\% respectivamente. 
QUADRO 11. Comprimento médio de raf́zes de cultivares de trigo medidos após 15 dias de crescimento em soluções nutritivas contendo quatro concentrações de manganês

\begin{tabular}{lcrrrrrrr}
\hline \multirow{2}{*}{ Cultivares } & \multicolumn{6}{c}{ Concentrações de manganês nas soluções em mg/litro } \\
\cline { 2 - 9 } & \multicolumn{2}{c}{0,11} & \multicolumn{2}{c}{300} & \multicolumn{2}{c}{600} & \multicolumn{2}{c}{1.200} \\
\hline & $\mathrm{mm}$ & $\%$ & $\mathrm{~mm}$ & $\%$ & $\mathrm{~mm}$ & $\%$ & $\mathrm{~mm}$ & $\%$ \\
IAC 162 & 145,6 & 100 & 129,9 & 84 & 117,6 & 81 & 75,4 & 52 \\
IAC 60 & 153,3 & 100 & 125,9 & 83 & 109,0 & 72 & 64,5 & 43 \\
BH 1146 & 178,6 & 100 & 128,9 & 73 & 95,8 & 54 & 37,4 & 21 \\
S. Cerros & 148,0 & 100 & 121,5 & 83 & 102,8 & 70 & 69,6 & 47 \\
\hline
\end{tabular}

No resultado do teste de moagem dos cultivares IAC 60 e IAC 162 - Quadro 12 - observou-se que seus rendimentos em farinha estão dentro do padrão, podendo ser comparáveis aos rendimentos dos trigos importados. Deve-se salientar que, devido às próprias características, os trigos produzidos no Brasil têm dificuldade durante a moagem, o que propicia sempre menor rendimento em farinha. Os resultados não demonstraram diferenças entre os dois novos cultivares em estudo.

QUADRO 12. Características de moagem dos cultivares IAC 60 (Centenário) e IAC 162 (Tuiuiú)

\begin{tabular}{llllll}
\hline Cultivares & R.F.T & R.F.G & R.F.F. & Perdas \\
\hline & & & $\%$ & & \\
\cline { 2 - 4 } IAC 60 & 71,28 & 18,91 & 8,79 & 1,02 \\
IAC 162 & 72,17 & 18,02 & 8,71 & 1,10 \\
\hline
\end{tabular}

R.F.T. = rendimento em farinha; R.F.G. = rendimento em farelo grosso; R.F.F. = rendimento em farelo fino.

Quanto às características físicas e ao teor de glúten úmido - Quadro 13 - o 'IAC 60', embora com teor menor do que o 'IAC 162', demonstrou qualidade de glúten superior, o que é mais interessante para a panificação. $O$ "falling number", que se relaciona com a atividade enzimática da farinha, mostrou valor bem melhor para o IAC 60 que para o IAC 162, que apresentou valor abaixo do considerado como limite (150 segundos). Este fator refletiu nas características de panificação, como se vê no quadro 17. Os valores mais recomendados para esta característica ('falling number") estão entre 300 e 350 segundos. Ficou expresso nos valores 
obtidos no amilógrafo - Quadro 14 - onde se verificou, por exemplo, um valor multo baixo de viscosidade máxima para o IAC 162, indicando a existência de grande atividade enzimática. Como resultado dessa atividade, obteve-se um pão com miolo excessivamente úmido, pois as enzimas amilolticas, agindo sobre o amido, transforma-o, deixando-o com excesso de água. Já o IAC 60 mostrou valor de viscocidade máxima a quente dentro dos valores usuais.

QUADRO 13. Características físicas da farinha dos cultivares IAC 60 e IAC162 comparadas à farinha comercial

\begin{tabular}{lccc}
\hline Cultivar & T.G.U. & Q.G. & F.N. \\
\hline IAC 60 & 33,5 & 13 & 538 \\
IAC 162 & 38,8 & 10 & 105 \\
Farinha comercial & 30,0 & 18 & 540 \\
\hline
\end{tabular}

T.G.U. = teor de glúten úmido; Q.G. = qualidade do glúten; F.N. = "falling number".

QUADRO 14. Características amilográficas da farinha de trigo dos cultivares IAC 60 e IAC 162

\begin{tabular}{lccc}
\hline Cultivar & T.G. & V.M. & T.V.M. \\
\hline & ${ }^{\circ} \mathrm{C}$ & U.B. & ${ }^{\circ} \mathrm{C}$ \\
IAC 60 & 57,0 & 1290 & 90 \\
IAC 162 & 54,7 & 40 & 90 \\
Farinha comercial & 60,0 & 1050 & 90 \\
\hline
\end{tabular}

T.G. = temperatura de gelatinização; V.M. = viscosidade máxima em umidade Brabender (U.B.); T.V.M. = temperatura de viscosidade máxima.

Com relação às características farinográficas - Quadro 15 - verificaram-se diferenças entre os cultivares. A resistência $(R)$ da massa preparada com a farinha obtida do 'IAC 60' foi superior à do 'IAC 162', indicando melhores características de manuseio durante o processamento do pão; isso foi corroborado pelo valor do abrandamento, que indica a perda de condição de resistência da massa à ação mecânica das pás da misturadeira após determinado período. Quanto aos demais parâmetros na curva farinográfica, praticamente não houve diferenças a salientar.

No quadro 16, pode-se observar outras características importantes da massa: a capacidade de produção e retenção de gás carbônico. Pelos dados obtidos neste teste, O 'IAC 162' teve comportamento um pouco superior no que se refere à característica de reter o gás carbônico produzido pela fermentação, parâmetro esse mais importante do que a própria produção de gás. 
QUADRO 15. Características farinográficas da farinha de trigo dos cultivares IAC 60 e IAC 162

\begin{tabular}{lccccr}
\hline Cultivar & T.D. & Abs. & E. & R. & A. \\
\hline & min. & $\%$ & $\min$. & $\min$. & U.B. \\
IAC 60 & 4,0 & 61,1 & 5,5 & 9,5 & 130 \\
IAC 162 & 3,5 & 61,9 & 2,0 & 5,5 & 240 \\
Farinha comercial & 5,0 & 65,0 & 3,0 & 8,0 & 50 \\
\hline
\end{tabular}

T.D. = tempo de desenvolvimento; Abs. = absorção de água; $E$. = estabilidade; $R$. = resistência; A. = abrandamento em unidade Brabender.

QUADRO 16. Características expansográficas da farinha de trigo dos cultivares IAC 60 e IAC 162

\begin{tabular}{llr}
\hline Cultivar & C.P. & Es. \\
\hline & $\mathrm{mm}$ & $\mathrm{mm}$ \\
IAC 60 & 60,0 & 60,0 \\
IAC 162 & 63,5 & 75,3 \\
Farinha comercial & 80,0 & 102,0 \\
\hline
\end{tabular}

C.P. = capacidade de produção de gás carbônico; Es. = estabilidade.

Os valores comparativos dos volumes especfficos dos pães - Quadro 17 indicaram que o 'IAC 162' comportou-se melhor, mas que, na contagem total, foi inferior ao 'IAC 60', devido ao seu baixo valor no "falling number", que resultou em miolo com características inadequadas.

QUADRO 17. Características dos pães obtidos com farinha dos cultivares IAC 60 e IAC 162 comparadas às da farinha comercial

\begin{tabular}{lcr}
\hline Cultivar & V.E.C. & C.T.C \\
\hline IAC 60 & 108,4 & 98,3 \\
IAC 162 & 112,5 & 94,4 \\
Farinha comercial & 100,0 & 100,0 \\
\hline
\end{tabular}

V.E.C. = volume específico comparativo; C.T.C. = contagem total comparativa. 


\section{CONCLUSÕES}

1. Os cultivares IAC 60 e IAC 162 não apresentaram diferenças entre si na produtividade de grãos e também em comparação às testemunhas Anahuac, IAC 24 e BH 1146, no Vale do Paranapanema, em condição de sequeiro, em diferentes tipos de solo quanto à acidez.

2. No Norte do Estado de São Paulo, os cultivares IAC 60 e IAC 162, com irrigação por aspersão, tiveram produtividade superior à dos controles Anahuac e IAC 24.

3. $O$ 'IAC 60' apresentou reação de suscetibilidade à ferrugem-do-colmo em casa de vegetação e o IAC 162, resistência.

4. $O$ 'IAC 60' demonstrou moderada suscetibilidade à ferrugem-da-folha e 0 'IAC 162', resistência, em condições de campo (sequeiro e irrigado).

5. Ambos os cultivares foram suscetiveis à helmintosporiose. Para a brusone, o 'IAC 162' foi resistente e o 'IAC 60', moderadamente suscetivel.

6. O 'IAC 60' apresentou tolerância ao alumínio tóxico e o 'IAC 162' moderada suscetibilidade. Ambos foram moderadamente sensiveis à toxicidade do ferro e tolerantes ao manganês.

7. Os novos cultivares apresentaram bom desempenho na panificação, sendo o IAC 162 levemente superior em relação ao volume especffico e inferior ao IAC 60 quanto às características internas e externas do pão.

\section{REFERÊNCIAS BIBLIOGRÁFICAS}

ALLARD, R.W. Principles of plant breeding. New York, John Wiley, 1960. 181p.

AMERICAN ASSOCIATION OF CEREAL CHEMISTS. Methods of analysis of AACC. St. Paul, 1969. n.p.

ARBEITSGEMEINSCHALT fuer Getreidefoschung Standart fuer geitreideund. Brot Detmold, Moritz Schaefer, 1971. 138p.

BÄR, W.H. Análise e avaliação do trigo e dos produtos derivados. Campinas, Instituto de Tecnologia de Alimentos, 1982. 154p.

CAMARGO, C.E. de O. Estudos de variedades de trigo para o Estado de São Paulo. Piracicaba, ESALQ, 1972. 102p. Tese (Doutorado).

—; FELIĆCIO, J.C.; FREITAS, J.G. de \& FERREIRA FILHO, A.W.P. Tolerância de trigo, triticale e centeio a diferentes níveis de ferro em solução nutritiva. Bragantia, Campinas, 47(2):295-304, 1988.

\& ROCHA JUNIOR, L.S. Trigo: tolerância ao alumínio em solução nutritiva. Bragantia, Campinas, 46(2):183-190, 1987.

\& OLIVEIRA, O.F. de. Tolerância de cultivares de trigo a diferentes níveis de manganês em solução nutritiva. Bragantia, Campinas, 42:65-78, 1983. 
CARVALHO, F.I.F. de. Genética quantitativa. In: FUNDAÇÃO CARGILL. Trigo no Brasil. Campinas, 1982. v.1, cap. 3, p.65-92.

CENTRO INTERNACIONAL DE MEJORAMIENTO DE MAIZ Y TRIGO (CYMMYT). Reseña de la Investigación 1984. México DF, 1985. 103p.

DUARTE, A.R. Tecnologia na agricultura: consideraçōes sobre a produtividade do trigo brasileiro. São Paulo, USP, 1974. 144p. Tese (Doutorado).

FELÍCIO, J.C.; BARROS, B. de C.; CAMARGO, C.E. de O. \& BÄR, W.H. Maracaí (IAC 17) e Xavantes (IAC 18): cultivares de trigo para o Estado de Săo Paulo. Bragantia, Campinas, 42:15-25, 1983.

-; CAMARGO, C.E. de O.; BARROS, B. de C. \& VITTI, P. Iguaçu (IAC 21) ө Araguaia (IAC 22): cultivares de trigo de sequeiro para o Estado de São Paulo. Bragantia, Campinas, 44(1):115-128, 1985.

; FERREIRA FILHO, A.W.P.; FREITAS, J.G. de; BARROS, B. de C. \& VITTI, P. Tocantins (IAC 23) e Tucuruí (IAC 24): novos cultivares de trigo. Bragantia, Campinas, 47(1):93-107, 1988.

; VITTI, P. \& GALLO, P.B. IAC 25 (Pedrinhas) e IAC 161 (Taiamã): novos cultivares de trigo. Bragantia, Campinas, 49(1):105-125, 1990.

— ; - FREITAS, J.G. de; FERREIRA FILHO, A.W.P.; BARROS, B. de C. \& CAMARGO, M.B.P. de. Avaliação de genótipos de irigo para a região do Vale do Paranapanema no quadriênio 1981-84. Bragantia, Campinas, 45(2):257-277, 1986.

IGARASHI, S.; UTIAMADA, C.M.; IGARASHI, L.C.; KAZUMA, A.H. \& LOPES, R.S. Pyricularia sp., em trigo: I. Ocorrência de Pyricularia sp. no Estado do Paraná. In: REUNIÃO NACIONAL DE PESQUISA DE TRIGO, 14., Londrina, 1986. p.57.

SCHRAMM, W.; FULCO, W.S.; SOARES, M.H.G. \& ALMEIDA, A.W.P. Resistência de cultivares de trigo em experimentação ou cultivo no Rio Grande do Sul, às principais doenças fúngicas. Agronomia Sulriograndense, Porto Alegre, 10(1):31-52, 1974.

SILVA, E.M.; LUCHIARI JÚNIOR, A.; GUERRA, A.F. \& GOMIDE, R.L. Recomendação sobre o manejo de irrigação em trigo para a região dos cerrados. In: REUNIÃO DA COMISSÃO NORTE-BRASILEIRA DE PESQUISA DE TRIGO, 10., Campinas, 1984. Brasília, EMBRAPA-CPAC, 1984. 60p.

TEIXEIRA, E.F. O trigo no Sul do Brasill. São Paulo, Lnotype, 1958. 300p.

VITTI, P.; LEITÃO, R.F. de F.; PIZZINATTO, A. \& PENTEADO, R.L.B. Preparo de uma farinha de milho integral e desengordurada e seu uso em produtos de panificação. Boletim do ITAL, Campinas, 17(4):451-467, 1980.

; MOREIRA, R.T. \& BÄR, W.H. Estudos tecnológicos do cultivar de trigo IAC 18. Boletim do ITAL, Campinas, 19(2): 183-194, 1982.

\& PIZZINATTO, A. Emprego da farinha de raspa de mandioca pré-gelatinizada em pão $\theta$ biscoito. Coletânea do ITAL, Campinas, 6:409-429, 1975. 